\title{
El Rol de la Inversión Pública en el Desempeño Económico Regional del Perú: 2001 - 2014
}

\author{
Melissa Castillo \\ Universidad de Chile
}

\section{Resumen}

Este estudio tiene como objetivo general analizar los efectos de la inversión pública en el nivel de producción - medido por el Valor Agregado Bruto (VAB) - de las regiones de Perú durante el periodo 2001 - 2014. Se realiza un análisis cuantitativo de tipo correlacional. Siguiendo la línea de las nuevas teorías de desarrollo regional se proponen tres modelos que intentan capturar: la importancia del capital público total, del capital público cuando se incluye una variable de desarrollo humano y de distintos tipos de capital público, al nivel de producción regional per cápita. La evidencia respalda la existencia de una relación positiva y significativa entre el capital público y el VAB per cápita regional. Además, se encuentra que la causalidad (en el sentido de Granger) es unidireccional en la mayoría de las regiones y fluye desde el capital público al VAB. Los resultados permiten hacer propuestas de política respecto al redireccionamiento de las inversiones públicas hacia sectores que tiendan a la acumulación de capital humano, en tanto las regiones presenten niveles incipientes de desarrollo.

Palabras clave: Desarrollo territorial, inversión pública, crecimiento económico.

The Public Investment Role in the Peru's Regional Economic Performance: 2001-2014

\begin{abstract}
The objective of this study is to analyze the effects of public investment on the level of production -measured by the Gross Value Added (GVA)- of the Peru's regions during the period 2001-2014. A quantitative analysis of correlational type is performed. Following the line of the new theories of regional development, three models are proposed that try to capture: the importance of the total public capital, of the public capital when it includes a variable of human development and of different types of public capital, to the regional production level per capita. The evidence supports the existence of a positive and significant relationship between public capital and the regional GVA regional per capita. In addition, it is found that causality (in the Granger sense) is unidirectional in most regions and flows from public capital to GVA. The results allow making policy proposals regarding the redirecting of public investments towards sectors that would tend towards the accumulation of human capital, if the regions present incipient levels of development.
\end{abstract}

Keywords: Territorial development, public investment, economic growth. 


\section{Crecimiento económico y desa- rrollo territorial}

El crecimiento económico es un aspecto fundamental del desarrollo ${ }^{1}$. La evidencia empírica sugiere que el crecimiento de los ingresos ha sido una fuente de reducción de la pobreza en distintos países; por ejemplo Agrawal (2007) cita los trabajos de Dollar y Kraay (2003), Deaton y Dreze (2001), Bhagwati y Srinivasan (2002) y Ravallion y Datt (2002). Siguiendo la línea de las nuevas teorías de desarrollo regional, en torno al reconocimiento de que "el espacio importa", es decir que los procesos innovativos y el crecimiento económico son fundamentalmente espaciales, las inversiones públicas deberían estar más orientadas hacia la acumulación del capital social y humano en las regiones, con el fin de mejorar su capacidad de innovación y la creación de condiciones para un proceso de auto refuerzo de desarrollo acumulativo (Uzbay y Lenger, 2011:11).

Perú es un país política y administrativamente unitario y descentralizado. Se caracteriza por ser una economía en vías de desarrollo que aspira a elevar los bajos estándares de vida de gran parte de su población y a gozar de un bienestar generalizado. En los últimos años, Perú ha tenido un desempeño económico destacable caracterizado por un entorno macroeconómico estable y altas tasas de crecimiento. Según estadísticas del Instituto Nacional de Estadística e Informática (INEI), entre los años 2001 y 2014, la economía peruana ha crecido a una tasa promedio anual per cápita de 4,3\%, una de las más altas a nivel de Latinoamérica. Gran parte de dicho dinamismo económico es atribuido al periodo expansivo del precio internacional de las materias primas que exporta.

En efecto, los ingresos vinculados a la minería e hidrocarburos han contribuido a incrementar sustancialmente los ingresos fiscales totales, lo cual ha incidido a su vez en un mayor ahorro fiscal y una reducción significativa de la deuda pública ${ }^{2}$. Aunado a ello, el Gasto No Financiero del Gobierno General creció en más del doble entre los años 2001 y 2014, debido, principalmente, a una mayor participación del gasto de capital, el cual pasó de un monto promedio de 3,0\% del PBI entre los años 2000-2007, a representar el 5,6\% del PBI en promedio durante

\footnotetext{
${ }^{1}$ Se entiende el desarrollo como un proceso multidimensional (económico, social, político, institucional, cultural, humano, entre otros) que posibilita cambios orientados a mejorar las condiciones de vida humana (Bertoni et al., 2011).

${ }^{2}$ Según estadísticas del MEF, el año 2014, el nivel de ahorro público total se encontraba por encima de $16 \%$ del PBI, siendo que el 4,7\% de PIB son recursos del Fondo de Estabilización Fiscal. Por su parte, la deuda pública se redujo de 48,2 \% del PBI en el 2001 a 20,1 \% del PBI en el 2014.
}

los últimos años (2008-2014).

Cabe indicar que los mayores flujos de inversión pública, en el periodo 2001-2014, se han dado en un contexto institucional marcado por la creación del Sistema Nacional de Inversión Pública, el cual busca una mayor eficiencia, sostenibilidad e impacto socioeconómico de las inversiones del gobierno; y por el impulso al proceso de descentralización. Es así que las inversiones realizadas en las regiones son formuladas, aprobadas y ejecutadas por el nivel nacional, a través de los ministerios sectoriales, así como por los gobiernos regionales y locales, de acuerdo a sus competencias.

En base a estos antecedentes, el presente estudio de caso pretende analizar, desde una perspectiva de crecimiento de la nueva economía regional, los efectos de la inversión pública en el nivel de producción per cápita regional. Asimismo, se busca aportar evidencia empírica que dé cuenta sobre el tipo de inversión pública sectorial que presenta mayores retornos (expresados en elasticidad producto - capital), aspecto que podría resultar útil en términos de direccionar el gasto público hacia las inversiones más rentables y que permitan un crecimiento regional sostenido.

\section{La inversión como determinante del crecimiento económico desde los en- foques neoclásico y endógeno}

Para los propósitos de la presente investigación se usará el término región en el sentido de "región de planificación" que corresponde a una unidad de control político o administrativo (Richardson, 1979). La ventaja de este enfoque es que existe una correspondencia directa entre las fronteras políticas y administrativas con los límites sobre los que los planificadores y hacedores de políticas diseñan e implementan políticas públicas (Dawkins, 2003: 133), lo cual se ajusta al contexto peruano.

Entendemos por inversión, al conjunto de desembolsos realizados por privados - individuos y empresas - y el gobierno, con la finalidad de acumular capital (Banco Mundial, 2013:138). En tal sentido, mientras el capital es una variable de stock, es decir hace referencia al conjunto de activos existentes en un territorio en un determinado momento del tiempo, la inversión es una variable de flujo, es decir varía a lo largo del tiempo.

Con respecto al capital público o de gobierno, Hansen (1965:5; citado por Uzbay y Lenger, 2011:3), hace una importante distinción entre "capital público fijo" y "capital público en infraestructura social". El "capital público fijo" implica inver- 
siones en infraestructura física con el fin de apoyar en forma directa actividades productivas de iniciativa privada. Por su parte, el "capital público en infraestructura social", implica inversiones en los seres humanos y en el medio ambiente ${ }^{3}$.

Aunque no necesariamente con un papel protagónico, la inversión aparece como factor explicativo del nivel de ingreso de los países en los trabajos de Solow (1956) y Swan $(1956)^{4}$. Éstos, desde una perspectiva neoclásica, parten suponiendo una economía cerrada y perfectamente competitiva, en la cual se produce un solo bien mediante el uso de dos factores: capital y trabajo. La versión básica de este modelo, se construye en base a dos ecuaciones: una función de producción que exhibe rendimientos constantes a escala y cuyos dos únicos factores productivos - capital y trabajo - se transan en mercados competitivos, son perfectamente sustituibles entre sí y presentan rendimientos marginales decrecientes; y, una ecuación que explica la acumulación de capital como resultado de la inversión actual (equivalente al ahorro bruto) la cual disminuye debido a la depreciación del stock de capital y el crecimiento poblacional.

Cass (1965) y Noriega (1965), citados por Noriega (2011:95), en base a los trabajos de optimización intertemporal de Ramsey (1928), realizan un aporte que puede considerarse un nexo analítico entre el crecimiento exógeno y endógeno. En el modelo hoy conocido como de "crecimiento óptimo de RamseyCass-Koopmans", el crecimiento del capital se deriva de la interacción entre familias que maximizan su utilidad en un horizonte infinito de vida, y empresas que maximizan sus beneficios.

Otro hito analítico en el desarrollo de las teorías explicativas del crecimiento, que incluyen a la inversión como factor determinante, son los trabajos de Romer $(1986,1990)$, mediante los cuales cobra relevancia una línea de teorías que explican el crecimiento endógenamente.

Romer (1986), presenta un modelo de crecimien-

\footnotetext{
${ }^{3}$ Siguiendo a Uzbay \& Lenger (2011:3), en la literatura más reciente encontramos el uso de "capital público fijo" como sinónimo de "infraestructura dura"; a ello le podemos agregar los términos "infraestructura de capital" (Gramlich, 1994) o "infraestructura económica" (Hernández, 2010:68). Asimismo, encontramos el uso de "capital público social" como similar de "infraestructura social" (Uzbay y Lenger, 2011: 3-4), la cual puede incluir tanto la infraestructura "blanda" y "dura" alrededor de los servicios y procesos que mejoran la capacidad social de las comunidades como educación, salud, bienestar social, arte y cultura, vivienda, empleo y entrenamiento, seguridad legal y pública, servicios de emergencia (Casey, 2005:3).

${ }^{4}$ Las primeras aproximaciones nos remontan a los desarrollos de Harrod (1939) y Domar (1946) de corte Keynesiano; no obstante, no es hasta el modelo de Solow - Swan que las teorías de creciendo económico cobran relevancia.
}

to a largo plazo en el que se asume que el conocimiento es un insumo de la producción que aumenta la productividad marginal. El modelo se basa en el marco propuesto por Arrow (1962) de "aprenderhaciendo" 5 para incorporar el cambio técnico como parámetro endógeno dentro de un modelo de equilibrio competitivo del crecimiento económico. La producción de bienes de consumo se modela con una función de producción que incluye el acervo de conocimientos y otros insumos. Esta función de producción asume rendimientos crecientes a escala en la producción de bienes de consumo, pero rendimientos de escala decrecientes en la producción de nuevos conocimientos, una característica que asegura la tratabilidad matemática (Dawkins, 2003:147).

Change (1990) presenta un modelo en el que el crecimiento es impulsado por el cambio tecnológico que surge de las decisiones de inversión hechas por agentes maximizadores de beneficios de manera intencionada. La característica distintiva de la tecnología endógena es que no es un bien convencional o bien público; es un bien no rival, parcialmente excluible; por lo tanto, no se puede mantener un escenario de equilibrio competitivo. En cambio, el equilibrio es uno de competencia monopolística. Una de las principales conclusiones es que el stock de capital humano determina la tasa de crecimiento.

\section{Nuevas teorías de desarrollo regional y el papel de la inversión pública}

El interés por incluir el factor espacial en las teorías de crecimiento económico surge como consecuencia de, por un lado, la importancia relativa que fueron cobrando las ciudades y regiones en los países europeos a partir de la década del setenta, a consecuencia del proceso de globalización, y de políticas de desregulación y ajuste estructural experimentadas (Stough et al., 2011: 3). A raíz del propio desarrollo teórico y trabajos empíricos, se reconoce que los procesos de innovación desencadenantes del crecimiento económico nacional son fundamentalmente de naturaleza espacial (Dawkins, 2003:132).

Sobre los aportes del modelo neoclásico a la comprensión del proceso de crecimiento y convergencia

\footnotetext{
${ }^{5}$ Arrow (1962) propone el marco de "aprender-haciendo", el cual está dentro de la tradición schumpeteriana con el argumento de que las empresas pueden obtener poder monopólico sobre los nuevos conocimientos a través de la experiencia en la producción interna. Las innovaciones se modelan como la disminución de los costos que son funciones de las inversiones anteriores de una empresa; así, si una empresa puede internalizar estos costos, pueden obtener una ventaja competitiva (Dawkins, 2003:147). La propuesta de Romer (1986) también es conocida como el modelo Romer-Arrow.
} 
regional, Alexiadis (2012) identifica dos: en primer lugar, cita a Armstrong y Taylor (2000) quienes señalan que el marco neoclásico llama la atención sobre la importancia de los factores de oferta en el proceso de crecimiento, es decir: la fuerza laboral, el stock de capital y el progreso técnico. En segundo lugar, el enfoque neoclásico de crecimiento regional lleva a la hipótesis de convergencia absoluta, que establece que, en el largo plazo, las economías regionales convergen al mismo nivel de "estado estacionario" de producción por unidad de trabajo, independientemente de las condiciones iniciales.

Por su parte, las nuevas teorías del desarrollo regional reconocen que el mecanismo de asignación de precios no necesariamente es eficiente en términos de asignación de recursos. En tal sentido se centran en la importancia de las economías de escala, los efectos de aglomeración y la difusión de conocimiento; e indican que el crecimiento económico tiende a ser más rápido en áreas que tienen un mayor stock de capital (mayor infraestructura), una población altamente educada y un entorno económico favorable a la acumulación de conocimiento; lo cual da lugar a patrones desiguales de desarrollo (Button, 1998; Uzbay y Lenger, 2011; Stough et al., 2011) ${ }^{6}$.

Un concepto importante sustenta a las nuevas teorías de desarrollo regional, se trata de la "causalidad circular acumulativa". Uzbay y Lenger (2011) citan a Myrdal (1970) quien sostiene que la "causalidad circular acumulativa" ayuda a explicar el hecho de que en una economía industrializada la inversión pública no limita su efecto a un aumento en el valor agregado de la producción; también induce a normas racionales de conducta y de organización, disciplina y habilidades empresariales vía efectos indirectos y externalidades que refuerzan aún más el desarrollo económico y transforman las estructuras sociales y económicas.

Uno de los principales aportes de la economía geográfica viene por el lado de las economías de aglomeración y localización, las cuales son importantes para explicar la agrupación espacial de los elementos clave para el crecimiento económico como son el capital humano y el progreso tecnológico; la implicación es que el desarrollo regional es altamente "dependiente de la trayectoria", y es desigual entre regiones y naciones (Porter, 1990; Krugman, 1991b, Cooke 2000; Martin y Sunley 2006; Schmutzler 1999; citados por Uzbay y Lenger, 2011:10).

Con respecto a investigaciones empíricas que ex-

\footnotetext{
${ }^{6}$ Stough et al. (2011) hacen hincapié, además, en la importancia que han cobrado los factores "intangibles" tales como el liderazgo, las instituciones, la creatividad, la innovación y el espíritu empresarial, los "intangibles" endógenos que pueden mejorar el rendimiento de las ciudades y regiones.
}

ploren la relación entre la inversión púbica y el desarrollo territorial, como uno de los antecedentes más emblemáticos tenemos los trabajos de Ashauer (1989a, 1989b), en los cuales se demuestra un fuerte rol positivo del capital público en el crecimiento económico de los Estados Unidos. La mayoría de los estudios posteriores apoyan un efecto positivo pero en algunos casos insignificante, dadas las diferencias en mediciones y enfoques empíricos usados (Shi, 2012:4).

Straub (2008), por su parte, hace una revisión de 140 especificaciones de 64 papers entre 1989 y 2007. Muestra que en el $63 \%$ de los casos analizados se encuentra una relación positiva y significativa entre infraestructura y alguna medida de desarrollo, mientras que en el $31 \%$ de los casos no se encuentra un efecto significativo y solo en el $6 \%$ restante se encuentra una relación negativa significativa $(19)^{7}$. No obstante, se hace notar que la diversidad de técni$\operatorname{cas}^{8}$, indicadores ${ }^{9}$, muestras ${ }^{10}$ y periodos de tiempo usados, hacen confuso el panorama cuando se trata de responder preguntas específicas de interés para los hacedores de política.

En esa línea, estudios recientes (Demurger, 2001; Mäki-Arvela, 2002; Drezgić, 2008; Wenjun y Jing, 2011; Shi, 2012; Mendoza Tolosa y Yanes Guerra, 2014) encuentran evidencia contundente a favor de la relación entre inversión pública (o algún proxy) y crecimiento económico. Sin embargo, también hay estudios que muestran evidencia mixta o estadísticamente no significativa (Cobacho et al., 2004; Arellano y Bond, 1991; Arellano y Bover, 1995; Uzbay y Lenger, 2011; González Páramo y López, 2003).

Las contribuciones al caso peruano, a nivel regional, se han enfocado principalmente en mostrar la relación entre diferentes tipos de infraestructura y crecimiento. Vásquez y Bendezú (2008) encuentran que la construcción de caminos tiene un efecto posi-

\footnotetext{
${ }^{7}$ Se señala que aunque puede existir la posibilidad de un sesgo de selección esta no es la regla, ya que se incluyen tanto documentos publicados como no publicados (Straub, 2008:19).

${ }^{8}$ La técnica macro-econométrica es la más frecuente: cerca de la mitad de los estudios que analiza usan la función de producción (49,3\%), seguida de las regresiones "crosscountry" $(21 \%)$, estimaciones en base a funciones de costo (9\%) y técnicas de contabilidad del crecimiento (3\%). Las especificaciones de nivel micro (data de hogares y firmas) suman aproximadamente el $18 \%$.

${ }^{9}$ Por ejemplo, mientras que en el periodo 1989 y 1999 el $72 \%$ de especificaciones usó alguna forma de capital público (inversión pública) y el $28 \%$ usó indicadores de capital físico; entre el 2000 y 2007 la relación se invirtió, solo el $24 \%$ usó variables de capital público y $76 \%$ usó alguna forma de indicadores físicos.

${ }^{10}$ Los resultados confirman que la relación positiva y significativa se da mayormente en muestras de países en desarrollo, aunque la diferencias es de $5 \%$ respecto a los países desarrollados. En muestras mixtas los resultados son menos precisos.
} 
tivo sobre el crecimiento económico de distintos departamentos. Urrunaga y Aparicio (2012) señalan que las infraestructuras de carreteras, electricidad y telecomunicaciones resultan relevantes para explicar las diferencias transitorias en el producto regional; asimismo, se encuentra evidencia que respalda la presencia de diferencias significativas en las repercusiones de las distintas infraestructuras en el producto per cápita de cada región.

Recientemente, Ponce (2013), tomando como marco el modelo propuesto por Barro y Sala-i Martin (1990) y usando la técnica econométrica para panel de datos dinámicos propuesta por Arellano y Bond (1991), devela que la inversión pública regional ha resultado un factor relevante en el crecimiento económico, aunque su contribución está por debajo de la que presenta la inversión privada. Una limitación de este estudio radica en que no considera la inversión pública de los niveles de gobierno nacional y local ejecutada en las regiones.

\section{Crecimiento económico regional e in- versión pública en el Perú: hechos es- tilizados}

La economía peruana se ha mostrado bastante dinámica en el periodo de estudio. Entre el 2001 y el 2014 el Producto Bruto Interno (PIB) per cápita creció a una tasa promedio anual real de 4,3\%, alcanzando un máximo el año 2007, con una tasa de $7,9 \%$. La crisis financiera internacional del $2008-$ 2009 , afectó el crecimiento $(-0,1 \%)$, sin embargo al año siguiente la economía se recuperó creciendo por encima del $7 \%$. Aunque en los últimos años el crecimiento se ha ralentizado, en parte producto de la caída de los precios internacionales de las materias primas que exporta, el PIB per cápita casi se duplicó en el periodo de estudio pasando de S/.8.335 en el 2001 a S/.15.152 nuevos soles en el 2014 .

No obstante, el crecimiento agregado esconde serias asimetrías latentes a nivel regional. Así, aunque todas las regiones han crecido en términos acumulados durante el periodo de análisis, se observa que aquellas regiones que empezaron más rezagadas, en términos de VAB per cápita en el 2001, son las que más han crecido; resaltan los casos de Cusco, Ayacucho y Amazonas, con tasas de crecimiento acumulado de $176,6 \%, 116,6 \%$ y $112,7 \%$ respectivamente. Por otro lado, las regiones que el año 2001 presentaban los mayores niveles de VAB per cápita, tuvieron tasas de crecimiento acumulado relativamente bajas; dentro de este grupo se encuentran Madre de Dios (7,7\%), Tacna (33,0\%), Pasco $(15,8 \%)$ y Moquegua $(39,5 \%)$. Pese a que este hecho nos da la idea de convergencia, vemos que el mayor crecimiento de las regiones más rezagadas ha sido insuficiente para lograr una nivelación respecto a las regiones con mayores VAB per cápita. Solo la región Cusco logró crecer lo suficiente para sobrepasar el VAB per cápita promedio del año 2014.

Luego, si comparamos los coeficientes de variabilidad (CV) del VAB per cápita de todas las regiones, observamos que este indicador se redujo en el periodo de estudio, pasando de 82,2\% el 2001 a $69,6 \%$ el 2014 , pese a la reducción, la variabilidad aún sigue siendo alta.

En cuanto a la inversión pública, observamos que el crecimiento acumulado real en el periodo de análisis sobrepasó el $155 \%$ en todas las regiones, sin embargo dicho crecimiento fue mas pronunciado en algunas regiones, por ejemplo Huancavelica, Ica y La Libertad presentan un crecimiento que sobrepasa el $700 \%$. Tal parece que las asimetrías se han ampliado, mientas que en el 2001 la brecha entre las regiones con mayor y menor nivel de inversión per cápita era de 5,3 veces; en el 2014 dicha brecha llega a 6,7 veces.

\section{Metodología, datos e hipótesis}

En esta investigación, se buscó probar dos hipótesis. La primera plantea que no existe una relación significativa entre la inversión pública y el VAB de la producción de las regiones del Perú en el periodo de estudio. La segunda, por su parte, plantea que las inversiones públicas en desarrollo social no son las que han tenido mayor incidencia en el VAB regional.

Con este objetivo, se desarrollaron tres modelos estadísticos. El primer modelo, parte desde una función de producción general tipo Cobb Douglas, y busca analizar los efectos del capital público en el crecimiento económico de las regiones del Perú en el periodo de estudio.

$$
\begin{array}{r}
L n y_{i t}=\delta_{0}+\alpha_{1} L_{n k p} p_{i t}+\alpha_{2} L n k p_{i t}+ \\
\beta L n l_{i t}+\epsilon_{i t}
\end{array}
$$

Puesto que el nivel de conocimiento/tecnología no puede ser observado directamente, es tratado como un coeficiente constante en la estimación y reporta la Productividad Total de Factores (PTF), la cual se denota por $\delta_{0}$. El stock de capital puede ser desagregado, denotando $\mathrm{kp}$ el acervo de capital privado y $\mathrm{kg}$ el acervo de capital público. $L$ es el factor trabajo; $i$ y $t$ indican la región y el año respectivamente. 
Figura 1: Perú: Producto Interno Bruto per cápita, 2001-2014

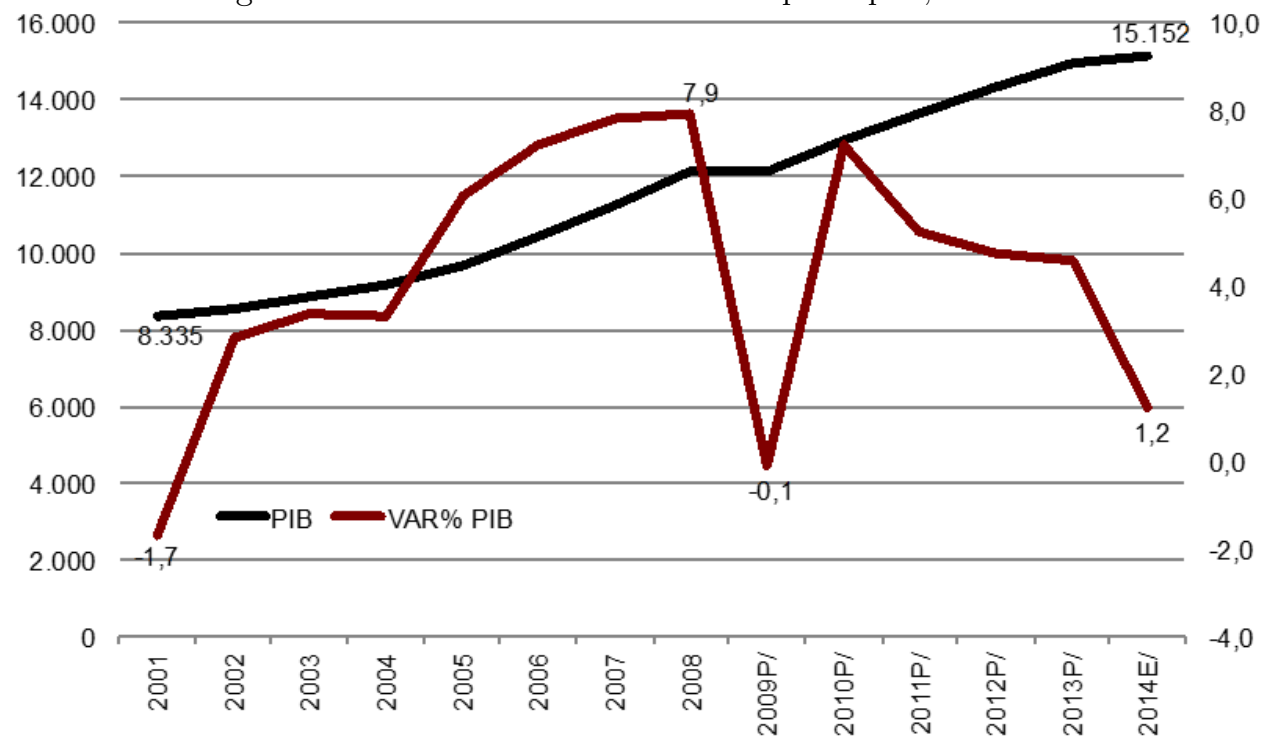

Nota: $\mathrm{P}=$ Preliminar; $\mathrm{E}=$ Estimado

Fuente: Elaboración propia sobre la base de datos de INEI

Figura 2: Perú: VAB per cápita según región, 2001y 2014

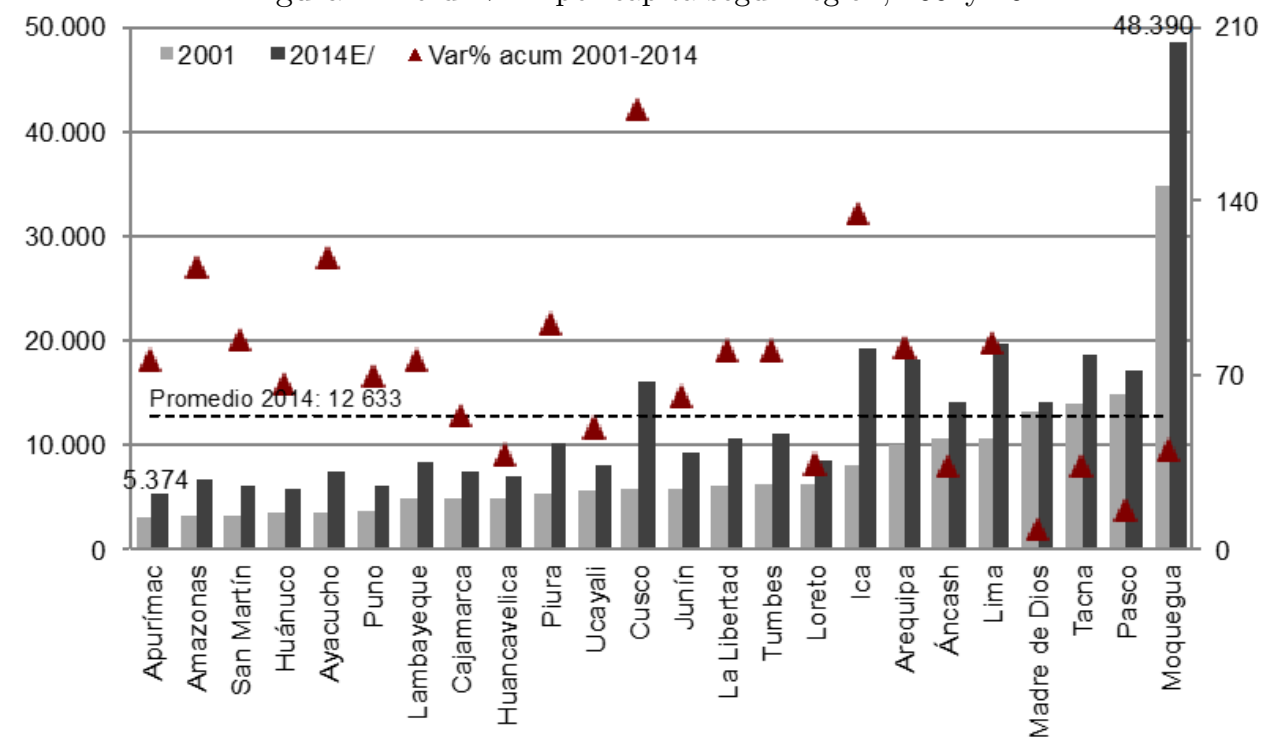

Fuente: Elaboración propia sobre la base de datos de INEI-SIRTOD. 
Figura 3: Perú: Inversión pública per cápita según región, 2001 y 2014

=2001 $=2014$ A Var\% Inv. pc

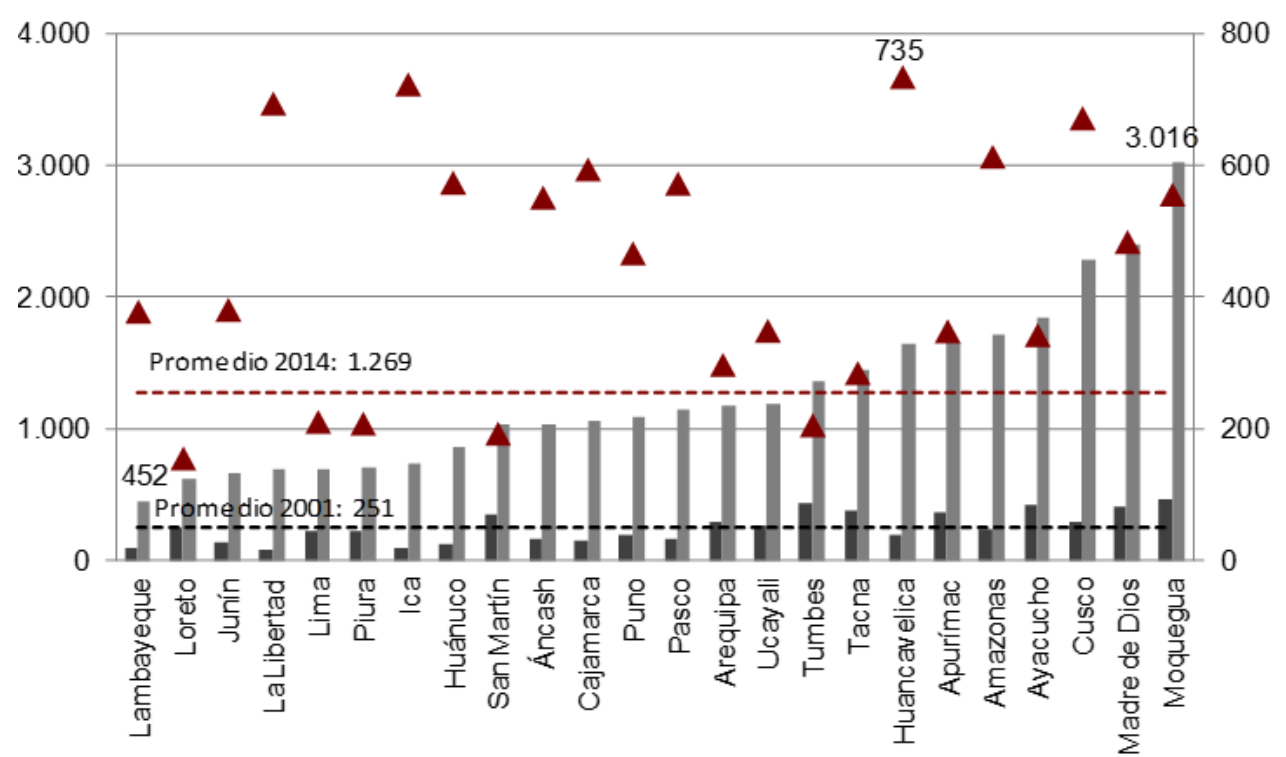

Fuente: Elaboración propia sobre la base de datos del MEF-Transparencia Económica e INEI.

Siguiendo a Uzbay y Lenger (2011), quienes plantean un modelo de crecimiento económico con capital humano, se introduce un canal adicional para analizar el efecto del desarrollo humano (DH) en el nivel de producción de las regiones. De acuerdo a la literatura revisada, la capacidad del capital público para generar efectos positivos sobre el nivel de producción está estrechamente relacionada con el perfil socio económico de las regiones. Es decir, las inversiones públicas tienen más éxito en el aumento del nivel de producción en las regiones donde se observan indicadores de desarrollo, como la educación y salud, más altos. Para probar esta afirmación, se construye un indicador de DH siguiendo la metodología del Índice de Desarrollo Humano (IDH) del Programa de las Naciones Unidad para el Desarrollo (PNUD), con variables aproximadas, disponibles para el periodo de estudio.

Aplicando logaritmos naturales y aproximando en términos per cápita, la función queda especificada econométricamente como

$$
\begin{array}{r}
L n y_{i t}=\delta_{0}+\alpha_{1} L_{k n k}+\alpha_{2} k g_{i t}+ \\
\beta L n h_{i t}+\epsilon_{i t} \\
o \\
L n y_{i t}=\delta_{0}+\alpha_{1} L n k p_{i t}+\alpha_{2} k g_{i t}+ \\
\beta_{1} L n l_{i t}+\beta_{2} L n D H_{i t}+\epsilon_{i t}
\end{array}
$$

Para responder a la pregunta sobre cuál de los sectores a los que se ha dirigido la inversión pública ha incidido más en el producto regional per cápita, se usa la siguiente ecuación:

$$
\begin{array}{r}
L n y_{i t}=\delta_{0}+\alpha_{1} L n k p_{i t}+\alpha_{2} L n k g-f p_{i t}+ \\
\alpha_{3} L n k g-s d_{i t}+\alpha_{4} L n k g-t c_{i t} \beta L n l_{i t}+ \\
\epsilon_{i t}
\end{array}
$$

donde $j$ es el sector al cual se dirige la inversión pública. Para efectos prácticos las inversiones se agrupan en infraestructura para el fomento productivo (fp), que incluye inversiones en las funciones agrícola, energía y minas, industria, comercio, servicios y turismo, pesca y trabajo; para el desarrollo social (ds), dentro de las que se incluyen las funciones asistencia y protección social, vivienda y desarrollo urbano, educación y cultura, salud y saneamiento; e infraestructura en transportes y comunicaciones (tc).

Al igual que Urrunaga y Aparicio (2012), se asume que no existen complementariedades entre los distintos tipos de acervo de capital, lo cual no es necesariamente cierto, ya que la existencia de infraestructura en transportes puede potenciar el capital en fomento productivo. Sin embargo, se asume la relación lineal dado que se procura analizar la contribución de cada tipo de capital al nivel de producción regional y estimar otro tipo de función podría distorsionar los resultados.

Para desarrollar la investigación, se utiliza un panel de datos para las 24 regiones del Perú durante el periodo 2001 - 2014. El periodo elegido se debe 
a la disponibilidad de información sobre inversiones públicas regionales; así, el panel está compuesto por 336 observaciones y es balanceado para cada especificación.

Según el modelo descrito, la variable dependiente es el producto regional, el cual es aproximado a través del Valor Agregado Bruto Departamental per cápita (como variable aproximada del PIB per cápita) en términos reales. Las series del VAB departamental tienen como fuente el Instituto Nacional de Estadística e Informática (INEI). Se cuenta con dos series: de 1999 al 2011 a precios constantes del año 1994 y del 2007 al 2014 a precios constantes del año 2007; por lo cual se procede a empalmar ambas series teniendo como referencia el año base más reciente. Por otro lado, dado que la información del VAB departamental de Lima incluye a Lima Metropolitana, Lima provincias y la Provincia Constitucional del Callao, se usa el mismo nivel de agregación para las demás variables (dependiente e intervinientes).

El capital se divide en público y privado. El capital público es la variable dependiente y se estima en base a la información sobre inversión pública usando el método de inventarios perpetuos. La información de las inversiones públicas regionales por funciones se obtiene a través de consultas de gasto mensual del portal web de Transparencia Económica del Ministerio de Economía y Finanzas (MEF). Solo en el caso de las inversiones realizadas en territorio regional por gobiernos locales, entre los años 2000 al 2008, se toma como fuente el Sistema de Contaduría Pública (SICON) del MEF, dado que dicha información no se encuentra disponible en el portal de Transparencia Económica. Cabe indicar, además, que a partir del año 2009 el MEF realizó un cambio en los clasificadores del gasto, por lo cual, los datos obtenidos tienen en cuenta las respectivas equivalencias entre el clasificador antiguo y el vigente.

Otro aspecto que se debe señalar respecto a la información de las inversiones públicas es la necesidad de deflactarlas para obtener las inversiones públicas a precios constantes del año 2007, para ello se usa el deflactor de la Formación Bruta del Capital Fijo Público total calculado en base a las series del INEI.

El capital privado, es una de las variables de control. Dado que no existe información disponible sobre inversiones o acervo de capital privado para regiones en Perú, se usa el consumo de electricidad industrial y comercial ${ }^{11}$ en términos per cápita como

\footnotetext{
${ }^{11}$ Esta aproximación fue usada por Uzbay y Lenger (2011) para el caso de Turquía, quienes citan a Moody (1974) como pionero en el uso de esta variable.
}

variable aproximada. Esta serie se obtiene a través de la página web del Organismo Supervisor de la Inversión en Energía y Minas (OSINERGMIN).

La otra variable de control es la fuerza de trabajo y puede aproximarse por medio de la PEA con nivel secundario, superior o ambos, o con los años de escolaridad promedio, que suele ser la más utilizada en los estudios similares. En el presente estudio se emplea la PEA con al menos nivel de educación secundario debido a la falta de estadísticas oficiales respecto de los años de escolaridad promedio de la PEA por región. Además, se maneja como indicador alternativo a la PEA en niveles, en caso de que la PEA con nivel secundario no sea significativa bajo algunas especificaciones. Ambos indicadores se estandarizan dividiéndolos entre la población de la región. La serie de PEA tiene como fuente la Dirección de Investigación Socio Económica Laboral (DISEL) y se obtiene a través del portal web del Ministerio de Trabajo y Promoción del Empleo.

La serie de población regional, requerida para expresar las variables en términos per cápita corresponde a estimaciones y proyecciones realizadas por el INEI y se obtiene a través del Sistema de Información Regional para la Toma de Decisiones (SIRTOD).

Para el caso de las especificaciones que incluyen como variable al capital humano, se usa un indicador de desarrollo humano (DH), el cual se construye siguiendo la metodología del IDH (PNUD, 2010, 2013). El indicador DH tiene en consideración solo dos dimensiones: salud y educación ${ }^{12}$. En el caso de salud, se usa como indicador la Esperanza de Vida al Nacer (EVN), la cual se obtiene a través del SIRTOD. Para educación se usan dos indicadores: tasa de conclusión secundaria para el grupo de edades de 17-18 (\% del total) y años de educación promedio de la población adulta (25-64 años); los cuales se obtienen a través del sistema de Estadística de la Calidad Educativa (ESCALE) del Ministerio de Educación (MINEDU) y del SIRTOD, respectivamente.

A continuación se muestra la correlación entre las variables en términos logarítmicos.

\section{Resultados}

Los modelos 1 y 2 buscan explicar la contribución del capital público agregado al VAB regional

\footnotetext{
${ }^{12}$ Cabe precisar que el IDH se basa en tres dimensiones: salud, educación y estándares de vida (medido a través del ingreso familiar per cápita); en este caso se excluye el indicador de ingresos para asegurar la independencia de la variable $\mathrm{DH}$ con la variable dependiente.
} 
Tabla 1: Correlación entre variables

\begin{tabular}{lccccccccc}
\hline \hline & 1 & 2 & 3 & 4 & 5 & 6 & 7 & 8 & 9 \\
\hline 1. Invab-pc & $1^{*}$ & - & - & - & - & - & - & - & - \\
2. lnkp-pc & $0,8^{*}$ & 1 & - & - & - & - & - & - & - \\
3. lnkg-pc & $0,36^{*}$ & 0,1 & 1 & - & - & - & - & - & - \\
4. lnkgfp-pc & $0,30^{*}$ & 0,09 & $0,73^{*}$ & 1 & - & - & - & - & - \\
5. lnkgds-pc & $0,5^{*}$ & $0,34^{*}$ & $0,81^{*}$ & $0,5^{*}$ & 1 & - & - & - & - \\
6. lnkgtc-pc & $0,14^{*}$ & $-0,06$ & $0,86^{*}$ & $0,42^{*}$ & $0,54^{*}$ & 1 & - & - & - \\
7. lnpea & $-0,2^{*}$ & 0,05 & $-0,42^{*}$ & $-0,5^{*}$ & $-0,2^{*}$ & $-0,36^{*}$ & 1 & - & - \\
8. lnpea1 & $-0,01$ & $0,19^{*}$ & $-0,35^{*}$ & $-0,49^{*}$ & $-0,01$ & $-0,32^{*}$ & $0,97^{*}$ & 1 & - \\
9. lndh & $0,7^{*}$ & $0,66^{*}$ & $0,18^{*}$ & 0,09 & $0,32^{*}$ & 0,07 & 0,09 & $0,3^{*}$ & 1 \\
\hline
\end{tabular}

Fuente: Elaboración propia sobre la base de estadísticas oficiales de Instituto Nacional de Estadística e Informática (INEI), Ministerio de Economía y finanzas (MEF), Organismo Supervisor de Energía y

Minas (OSIPTEL), Ministerio de Trabajo y Promoción del Empleo (MTP); 2015.

(todas las variables están expresadas en términos logarítmicos y per cápita). El modelo 1 es una función de producción tipo Cobb-Douglas simple; en ésta el VAB depende del capital público, el capital privado y la PEA educada (con al menos nivel secundario).

De entre los modelos estáticos más básicos, se obtiene que el modelo econométrico de Efectos Fijos (en adelante EF) es el más robusto una vez realizadas las correcciones por autocorrelación y heterocedasticidad (no se detecta presencia de correlación contemporánea) mediante la técnica de Errores Estándar Corregidos para Panel de Datos (en adelante PCSE). Todos los coeficientes resultan ser altamente significativos y con los signos esperados. Alternativamente, el modelo 1 se estima mediante Coeficientes Aleatorios (en adelante RCM), el cual también resulta significativo en su conjunto.

Como se aprecia en la Tabla 2, el coeficiente del capital público es muy parecido tanto con FE como con RCM, siendo aproximadamente de 0,19. Ello indica que ante el aumento de $1 \%$ en el capital público per cápita; el VAB percápita regional se incrementa en aproximadamente $19 \%$.

El modelo 2 incluye, en lugar de la PEA educada, la PEA total y el indicador de Desarrollo Humano (DH) ajustado, con la finalidad de analizar el efecto del DH en el nivel de producción de las regiones. Una vez más se obtiene que el mejor modelo es el FE estimado mediante PCSE corrigiendo por autocorrelación y heterocedasticidad. La estimación de RCM para este modelo también resulta significativa en su conjunto. El coeficiente del capital público es muy similar tanto con FE como con RCM y no varía mucho respecto a la ecuación 4, siendo de 0,21 aproximadamente. El coeficiente del DH resulta altamente significativo siendo de 0,37 con FE y de 0,60 con RCM; la diferencia entre ambas estima- ciones no nos permite concluir sobre la robustez de este parámetro.

Por otro lado, el modelo 3 estima la contribución de diferentes tipos de capital público al VAB regional. El mejor modelo resulta ser el de FE estimado con PCSE mediante el cual se corrigen los problemas de autocorrelación y heterocedasticidad. Solo el coeficiente del capital público en desarrollo social resulta con el signo esperado y es significativo, siendo de 0,18. El capital para el fomento productivo tiene el signo esperado, pero no es significativo, mientras que el capital público en transportes y comunicaciones tiene signo contrario y es no significativo al $95 \%$ de confianza.

Los coeficientes elevados nos hacen sospechar que éstos pueden estar sesgados por la endogeneidad entre el VAB y el capital público. Es por esto que, como alternativa, se realiza la Prueba de Causalidad de Granger ${ }^{13}$. Se testean dos hipótesis nulas:

- H0: El capital público per cápita no causa (en el sentido de Granger) el VAB per cápita. Si H0 se rechaza se puede interpretar como que cambios en el capital público per cápita preceden a cambios en el VAB per cápita.

- H0: El VAB per cápita no causa (en el sentido de Granger) al capital público per cápita. Si H0 se rechaza se puede interpretar como que cambios en el VAB cápita preceden a cambios en el capital público per cápita.

Para la mayoría de las regiones, la evidencia in-

\footnotetext{
${ }^{13} \mathrm{La}$ noción de Causalidad de Granger es una formalización de la idea de que el futuro no puede causar el pasado, esta está definida en términos de poder predictivo; es decir, una variable $\mathrm{X}$ causa una variable $\mathrm{Y}$, si los valores presentes de $\mathrm{Y}$ se pueden predecir mejor con los valores pasados de $\mathrm{X}$ que sin usar estos últimos (Munoz y Vindas, 1995).
} 


\begin{tabular}{|c|c|c|c|c|c|c|}
\hline Variable & FE PC SE & $\mathrm{RCM}$ & FE PCSE & $\mathrm{RCM}$ & FE PCSE & RCM \\
\hline Inkg_pc & $0.197^{\star \star \star *}$ & $0.193^{\star \star \star \star}$ & $0.206^{* \star *}$ & $0.208^{\star * *}$ & & \\
\hline Inkp_pc & $0.081^{\star * \pi *}$ & $0.171^{\text {*k*}}$ & $0.088^{\star * *}$ & $0.157^{\star \star *}$ & $0.071^{\star \star \star *}$ & $0.164^{* *}$ \\
\hline Inkgfp_pc & & & & & 0,014 & $-0,077$ \\
\hline Inkgds_pc & & & & & $0.179^{\star \star \star *}$ & 0,162 \\
\hline Inkgtc_pc & & & & & $-0,006$ & $-0,02$ \\
\hline Inpea1_pc & $0.367^{\star \star \star *}$ & $0.527^{\star \star \star *}$ & & & $0.329^{\star \star * *}$ & $0.371^{\star \star \star \star}$ \\
\hline Inpea_pc & & & $0.378^{\star \star \star *}$ & $0.416^{*}$ & & \\
\hline Indh & & & $0.370^{\star \star \star \star}$ & $0.606^{* * *}$ & & \\
\hline Áncash & $0.757^{\star \star \star \star}$ & & $0.791^{\star \star \star \star}$ & & $0.705^{\star \star \star \star}$ & \\
\hline Apurimac & $-0.250^{* * *}$ & & $-0221^{\star \star \star \star}$ & & $-0.206^{* * *}$ & \\
\hline Arequipa & $0.698^{\star \star \star *}$ & & $0.776^{\star \star \star}$ & & $0.750^{\star \star \star \star}$ & \\
\hline Ayacucho & 0,006 & & 0,045 & & $-0,002$ & \\
\hline Cajamarca & $0.326^{\star \star \star *}$ & & $0.282^{\star \star \star}$ & & $0.282^{\star \star \star *}$ & \\
\hline Cusco & $0.402^{* \star \star *}$ & & $0.446^{* \star *}$ & & $0.413^{\star * * *}$ & \\
\hline Huancavelica & 0,07 & & 0,081 & & $-0,021$ & \\
\hline Huánuco & 0,05 & & 0,068 & & $-0,06$ & \\
\hline Ica & $0.685^{\star \star \star *}$ & & $0.776^{\star \star \star *}$ & & $0.620^{* \star *}$ & \\
\hline Junín & $0.243^{\star \star}$ & & $0.312^{* * *}$ & & 0,204 & \\
\hline La Libertad & $0.502^{\star \star \star *}$ & & $0.562^{* \star \star *}$ & & $0.468^{\star * \star *}$ & \\
\hline Lambayeque & $0.248^{\star \star \star *}$ & & $0.317^{\star \star \star \star}$ & & $0.189^{*}$ & \\
\hline Lima & $0.777^{\star \star \star \star}$ & & $0.904^{\star \star * *}$ & & $0.669^{\star * \star *}$ & \\
\hline Loreto & $0.377^{\star \star \star \star}$ & & $0.488^{\star \star \star \star}$ & & $0.250^{*}$ & \\
\hline Madre de Dios & $0.811^{\star \star \star \star}$ & & $0.921^{\star \star \star *}$ & & $0.911^{\star \star \star \star}$ & \\
\hline Moquegua & $1.537^{\star \star \star \star}$ & & $1.583^{\star \star \star \star}$ & & $1.542^{* * *}$ & \\
\hline Pasco & $1.004^{\star \star \star *}$ & & $1.061^{\star * \star *}$ & & $0.930^{\star \star \star *}$ & \\
\hline Piura & $0.354^{\star \star \star *}$ & & $0.399^{\star \star \star \star}$ & & $0258^{\star \star \star \star *}$ & \\
\hline Puno & $-0.163^{\star \star *}$ & & $-0,12$ & & $-0.146^{\star \star}$ & \\
\hline San Martín & $-0,084$ & & $-0,037$ & & $-0,004$ & \\
\hline Tacna & $0.828^{\star \star \star *}$ & & $0.916^{\star \star \star}$ & & $0.809^{\star \star \star}$ & \\
\hline Tumbes & $0.242^{\star \star \star \star}$ & & $0.326^{\star \star \star *}$ & & $0.170^{*}$ & \\
\hline Ucayali & $0.164^{\star \star}$ & & $0.285^{\star \star \star \star}$ & & 0,117 & \\
\hline cons & $7.568^{\star \star \star}$ & $8.192^{\star \star \star}$ & $7.437^{\star \star \star}$ & $8.038^{* * *}$ & $7.835^{\star \star \star}$ & $9.169^{\star \star \star}$ \\
\hline $\bar{N}$ & 336 & 336 & 336 & 336 & 336 & 336 \\
\hline r2 & 0,996 & & 0,996 & & 0,996 & \\
\hline chi2 & 4182,266 & 123,387 & 4137,779 & 120,121 & 5117,022 & 46,189 \\
\hline
\end{tabular}

dica que la causalidad (en el sentido de Granger) es unidireccional y va del capital público al VAB ${ }^{14}$. Lo mismo es válido tanto para el capital público agregado como para el desagregado por tipos. Lo anterior nos permite robustecer los resultados de las estimaciones realizadas mediante modelos estáticos.

Aun así, es importante tener en cuenta lo que señala Drezgić (2008) respecto a tener cuidado con la magnitud de los coeficientes, ya que estos suelen ser sensibles a la muestra de las series de tiempo usadas. Ello implica que los resultados son válidos para el periodo de estudio y deben ser tomados con cautela si se trata de hacer predicciones.

Lo expuesto hasta este punto aporta evidencia a favor de una relación positiva y significativa entre el VAB regional y el capital público (que resulta de la acumulación de las inversiones públicas) para el caso peruano. El coeficiente obtenido para el capital público per cápita -entre 0,19 y 0,21-indica que la

\footnotetext{
${ }^{14}$ En 16 regiones la causalidad en el sentido de Granger fluye del capital público al VAB; en 3 regiones (Cajamarca, Madre de Dios y San Martin) no existe evidencia de causalidad en ningún sentido; mientras que en 4 regiones (Apurímac, Huancavelica, Huánuco y Junín) la causalidad en el sentido de Granger va del VAB al capital público.
}

inversión pública juega un papel muy importante en el desempeño económico de las regiones.

Asimismo, los resultados del modelo 3 sugieren evidencia a favor de que las inversiones públicas en desarrollo social son las que han tenido mayor incidencia en el VAB regional. En efecto, el único coeficiente que resulta significativo es el de capital público para el desarrollo social.

Resultados de estudios previos en los que se estima la elasticidad producto de distintos tipos de capital o infraestructura, no permiten llegar a un consenso respecto a la significancia en los párametros de los tipos de capital, así como tampoco sobre las magnitudes. Drezgić (2008), mediante FE, estima elasticidades de 0,04 y 0,08 para el capital público físico y social, respectivamente. Shi (2012), mediante FE dinámicos, encuentra que solo el coeficiente de las inversiones en electricidad es significativo con un valor de 0,28 , mientras que el coeficiente de las inversiones en carreteras tiene signo contrario $(-0,08)$ y es no significativo. Para el caso peruano, Vásquez y Bendezú (2008), mediante técnicas de cointegración, estiman una elasticidad producto de la infraestructura vial en 0,22; Urrunaga y Aparicio 


\begin{tabular}{|c|c|c|c|c|c|c|c|c|}
\hline Variable & $\begin{array}{c}\text { Causalidad } \\
\text { de kg a } \\
\text { vab }\end{array}$ & $\begin{array}{c}\text { Causalidad } \\
\text { de vab a } \\
\text { kg }\end{array}$ & $\begin{array}{c}\text { Causalidad } \\
\text { de kgfp a } \\
\text { vab }\end{array}$ & $\begin{array}{l}\text { Causalidad } \\
\text { de vab a } \\
\text { kgfp }\end{array}$ & $\begin{array}{c}\text { Causalidad } \\
\text { de kgds a } \\
\text { vab }\end{array}$ & $\begin{array}{c}\text { Causalidad } \\
\text { de vab a } \\
\text { kgds }\end{array}$ & $\begin{array}{c}\text { Causalidad } \\
\text { de kgtc a } \\
\text { vab }\end{array}$ & $\begin{array}{c}\text { Causalidad } \\
\text { de vab a } \\
\text { kgtc }\end{array}$ \\
\hline Amazonas & $0.661^{\star \star *}$ & $0.190^{*}$ & $0.796^{\star \star \star}$ & 0,296 & $0.4568^{\star *}$ & $0.262^{* \star}$ & $0.680^{\star \star *}$ & $0.139^{*}$ \\
\hline Áncash & $0.468^{\star \star}$ & 0,049 & $0.495^{\star \star *}$ & 0,078 & $0.468^{* *}$ & 0,039 & $0.432^{\star *}$ & 0,047 \\
\hline Apurimac & 0,033 & $0.871^{\star \star \star \star}$ & 0,289 & $1.240^{\star \star \star \star}$ & 0,100 & $0.315^{\star \star \star \star}$ & 0,349 & $0.860^{\star \star \star \star}$ \\
\hline Arequipa & $1.036^{* * *}$ & $-0,146$ & $1.028^{* * *}$ & $-0,129$ & $1.087^{* * *}$ & $-0,074$ & $0.959^{* \pi *}$ & $-0,198$ \\
\hline Ayacucho & $1.136^{\star * *}$ & $-0,185$ & $0.983^{* * *}$ & $-.5730^{\star}$ & $1.124^{\star \star * *}$ & $-0,081$ & $1.134^{* \star *}$ & $-0,077$ \\
\hline Cajamarca & 0,543 & 0,119 & 0,450 & 0,171 & 0,534 & 0,099 & $0.717^{\star}$ & 0,065 \\
\hline Cusco & $1.115^{\star \star * *}$ & $-0,065$ & $0.950^{* *}$ & 0,105 & $0.797^{\star}$ & 0,182 & $1.149^{* * *}$ & $-0,101$ \\
\hline Huancavelica & 0,023 & $0.393^{\star \star}$ & $0.959^{\star \star *}$ & 0,090 & 0,168 & $0.271^{\star *}$ & $0.577^{\star \star}$ & $0.148^{*}$ \\
\hline Huánuco & 0,005 & $0.552^{\star \star \star *}$ & 0,246 & $0.783^{\star \star \star}$ & $-0,077$ & $0.434^{\star \star \star *}$ & 0,169 & $0.554^{\star \star *}$ \\
\hline Ica & $1.110^{* \star *}$ & $-0,097$ & $1.081^{\text {***}}$ & $-0,244$ & $1.117^{* * *}$ & $-0,067$ & $1.096^{* * *}$ & $-0,123$ \\
\hline Junín & 0,296 & $0.336^{*}$ & $0.724^{*}$ & 0,209 & $0.571^{\star}$ & 0,178 & $-0,130$ & $0.555^{* * *}$ \\
\hline La Libertad & $1.040^{* * *}$ & $-0,050$ & $1.007^{\star \star \star *}$ & $-0,213$ & $1.034^{* * *}$ & $-0,026$ & $0.988^{* * *}$ & $-0,003$ \\
\hline Lambay eque & $1.052^{* \star *}$ & 0,000 & $1.130^{* \star *}$ & $-0,168$ & $1.008^{* *}$ & 0,022 & $0.972^{\text {** }}$ & 0,096 \\
\hline Lima & $1.068^{* * *}$ & $-0,164$ & $0.960^{* \star *}$ & 1,077 & $0.977^{* \star *}$ & $-0,349$ & $1.104^{\text {} * \star}$ & $-0,066$ \\
\hline Loreto & $0.736^{* *}$ & 0,301 & 0,063 & $0.884^{\star \star \star}$ & $0.686^{*}$ & 0,224 & $0.986^{\star \star *}$ & 0,065 \\
\hline Madre de Dios & 0,585 & $-0,023$ & 0,262 & -1.072 & 0,469 & 0,029 & 0,586 & $-0,017$ \\
\hline Moquegua & $0.682^{\star \star *}$ & 0,009 & $0.683^{\star \star *}$ & 0,015 & $0.678^{* *}$ & 0,008 & $0.678^{* \star}$ & 0,008 \\
\hline Pasco & $0.695^{\star \star}$ & $-0,013$ & $0.690^{* *}$ & $-0,032$ & $0.687^{\star \star \star}$ & $-0,011$ & $0.705^{\star \star}$ & $-0,011$ \\
\hline Piura & $1.129 * * *$ & $-0,189$ & $1.153^{* \star *}$ & $-0,369$ & $1.140^{* \star *}$ & $-0,152$ & $1.079^{* \star *}$ & $-0,130$ \\
\hline Puno & $1.143^{\star * *}$ & $-0,091$ & $1.173^{* \star *}$ & $-0,099$ & $0.878^{* *}$ & 0,079 & $1.083^{\star \star \star *}$ & $-0,075$ \\
\hline San Martín & $0.740^{* \star}$ & 0,389 & 0,552 & 0,809 & 0,554 & 0,233 & $0.828^{* * *}$ & 0,447 \\
\hline Tacna & 0,561 & 0,125 & $0.796^{\star \star}$ & 0,194 & 0,419 & $0.096^{*}$ & 0,606 & 0,081 \\
\hline Tumbes & $0.741^{\star}$ & 0,175 & $1.002^{* \pi *}$ & $-0,015$ & $0.734^{*}$ & 0,170 & 0,607 & 0,176 \\
\hline Ucayali & $0.764^{\star \star * *}$ & 0,065 & $0.924^{\star \star \star *}$ & 0,005 & $0.889^{\star * *}$ & 0,018 & $0.697^{\star \star *}$ & 0,065 \\
\hline $\begin{array}{l}\text { Nro. casos } \\
\text { (causalidad } \\
\text { unidireccional) }\end{array}$ & 16 & 4 & 17 & 3 & 16 & 4 & 16 & 3 \\
\hline
\end{tabular}

(2012), con técnicas para panel de datos estático, estiman elasticidades producto de 0,$12 ; 0,07$ y 0,03 para infraestructura en electricidad, telecomunicaciones y transportes, respectivamente.

Por otro lado, el hecho de que las ecuaciones estimadas mediante RCM sean significativas en su conjunto, para todos los casos, aporta evidencia a favor de que la inversión pública total y los distintos tipos de inversión, en lo particular, tienen efectos disímiles en el desempeño económico de las regiones. En las estimaciones realizadas, las pruebas $C h i^{2}$ permiten rechazar la hipótesis nula de que los efectos aleatorios son todos cero para cada modelo, por lo tanto, es permisible que los coeficientes varíen entre regiones, es decir que el capital público incide de manera diferenciada entre regiones.

Al respecto, la Tabla 3 reporta las estimaciones detalladas por región mediante RCM. En la Modelo 1 , el coeficiente del capital público resulta significativo y con el signo esperado en 15 de las 24 regiones, con valores que van de 0,11 (Lima) a 0,41 (Huancavelica). Cuando incluimos el Indicador de Desarrollo Humano (modelo 2), el coeficiente del capital público es significativo en 17 regiones, estando entre 0,16 (Ayacucho, Ucayali y Lambayeque) y 0,41 (Amazonas). Llama la atención que en este modelo el coeficiente para Pasco es de -0,10 (significativo solo al $90 \%$ de confianza); es decir que ante el aumento de $1 \%$ en el capital público per cápita, el VAB per cápita de esta región se reduce en $10 \%$, lo que implica que el capital público adicional no es productivo en esta región.

En la misma línea, cuando analizamos la elasticidad producto de los distintos tipos de capital (Modelo 3), vemos que el coeficiente de fomento productivo solo es significativo en cuatro regiones (Amazonas, Ica, Moquegua y Puno), siendo negativo para todas ellas, excepto en Puno $(0,25)$. El capital en desarrollo social resulta significativo en 14 casos, con coeficientes positivos que oscilan entre 0,21 (Huancavelica) y 0,68 (Cusco); en los casos de Lima y Madre de Dios la elasticidad producto de este tipo de capital es negativa $(-0,45$ y $-0,55$, respectivamente). Por su parte, el capital en transporte y comunicaciones resulta significativo en 9 regiones, siendo positivo solo en cuatro de ellas (Junín: 0,29; Lima: 0,14; Pasco: 0,22 y Tumbes, 0,26).

Respecto a los criterios y principios sobre los cuales se basan las inversiones, la incidencia de los distintos tipos de inversión en el crecimiento debería tender a variar según el estadio de desarrollo de las unidades territoriales. Aunque Perú exhibe niveles muy desiguales en el VAB per cápita entre sus regiones, la mayoría de ellas se encuentra en niveles incipientes de desarrollo. Haciendo comparaciones con Estados Unidos, el PIB per cápita estatal del 2014 fluctúa entre de US\$ 31.551 (Mississipi) y US\$ 66.160 (Alaska). Para no ir tan lejos, en Colombia el PIB per cápita ajustado por paridad de poder de compra (PPA) va de US\$ 3.887 (Vaupés) 
Tabla 4: Estimaciones de coeficientes diferenciados por región mediante RCM.

\begin{tabular}{|c|c|c|c|c|c|c|c|c|c|c|c|c|c|c|c|}
\hline \multirow{2}{*}{ Región } & \multicolumn{4}{|c|}{ Modelo 1} & \multicolumn{5}{|c|}{ Modelo 2} & \multicolumn{6}{|c|}{ Modelo 3} \\
\hline & Inkg_pc & Inkp_pc & Inpea1_pc & cons & Inkg_pc & Inkp_pc & Inpea_pc & Indh & cons & Inkgfp_pc & Inkgds_pc & Inkgtc_pc & Inkp_pc & Inpea1_pc & cons \\
\hline Amazonas & $0.384^{z z z}$ & 0,067 & $0.581^{z+2 x}$ & $6.275^{\text {taz }}$ & $0.418^{\text {tatz }}$ & 0,068 & $0.617^{*}$ & $0.836^{2}$ & $6.05^{\text {kz* }}$ & $-0.732^{2 * t z}$ & $0.47^{* 2 \approx}$ & 0,102 & $0.093^{\text {taxt }}$ & 0,083 & $9.890^{\text {zazk }}$ \\
\hline Áncash & $-0,042$ & $0.255^{x z}$ & $0.622^{* x *}$ & $10.608^{2 \times x}$ & 0,003 & $0.309^{\text {rx }}$ & 0,483 & $0.698^{\text {tx }}$ & $10.138^{\text {xxx }}$ & $-0,444$ & 0,078 & 0,079 & $0.258^{\text {zx }}$ & 0,455 & $12.139^{\mathrm{xz}}$ \\
\hline Apurimac & 0,478 & 0,002 & 0,429 & 4,763 & 0,471 & 0,054 & 0,200 & 0,073 & 4,553 & $-0,205$ & 0,171 & 0,340 & $-0,028$ & 0,513 & 6,434 \\
\hline Arequipa & $0.166^{* z z}$ & $0.304^{\approx \pi x}$ & $0.482^{*}$ & $8.626^{* \pi z}$ & $0.227^{* \pi x}$ & $0.306^{x \times x}$ & $-0,050$ & $0.842^{2 k}$ & $7.882^{2 \times x}$ & $-0,048$ & $0.242^{\text {zx }}$ & $-0.254^{\text {zz }}$ & $0.214^{z k z}$ & 0,298 & $10.42^{\mathrm{x*x}}$ \\
\hline Ayacucho & $0.213^{\text {tz }}$ & $0.263^{* x *}$ & $0.466^{* \pi}$ & $8.106^{\star \star x z}$ & $0.161^{*}$ & $0.319^{\text {z*k }}$ & 0,220 & $0.507^{*}$ & $8.522^{\text {**k }}$ & 0,024 & $0.320^{*}$ & $-0,089$ & $0.282^{* * z}$ & 0,226 & $7.813^{\text {tkx }}$ \\
\hline Cajamarca & $0.200^{\text {tz*zz}}$ & $0.133^{ \pm x *}$ & $-0,304$ & $6.872^{\star * x}$ & $0.174^{2 * x}$ & $0.103^{\text {tz }}$ & $-0,144$ & $-0,180$ & $7311^{\text {knk}}$ & 0,118 & 0,007 & 0,087 & $0.117^{\star x *}$ & $-0,214$ & $7.136^{\text {tzk}}$ \\
\hline Cusco & $0.330^{z z z}$ & 0,125 & $1.376^{\star \star \pi k}$ & $8.068^{x * x}$ & 0.378 & 0,085 & $1.063^{\operatorname{zxz}}$ & $1.597^{\star x x}$ & 7.47 & $-0,232$ & & $-0,098$ & 0,067 & $0.866^{\text {zxz }}$ & $7.681^{\mathrm{mx}}$ \\
\hline Huancavelica & 0.413 & 0,026 & $-0,151$ & 4.94 & & 0,017 & & $-0,037$ & $5227^{2 \pi k}$ & 0,072 & & 0,129 & 0,008 & $-0,153$ & $5.413^{\text {zkx }}$ \\
\hline Huánuco & $0.360^{\mathrm{xzz}}$ & $-0,004$ & $0.273^{x *}$ & $5.991^{\pi x x}$ & $0.387^{\approx \pi x}$ & 0,003 & $0.586^{*}$ & 0,220 & $5932^{2 \times x}$ & 0,088 & $0.244^{* x}$ & $-0,012$ & $-0,009$ & $0.254^{\text {zz }}$ & $6.578^{x \approx x}$ \\
\hline Ica & $0.212^{2 x z}$ & 0,018 & $1.853^{\star x k \pi}$ & $9.589^{2 \pi x}$ & $0.209^{z x x}$ & $-0,097$ & $2.435^{\text {zak }}$ & $1.349^{z * z}$ & $10.113^{\approx \times x}$ & $-0.627^{* \pi}$ & $0.388^{8 * z}$ & $-0,061$ & 0,042 & $1.395^{\text {zkz }}$ & $12.282^{2 x *}$ \\
\hline Junin & $0.286^{z \pi z}$ & $0.134^{* z}$ & $0.52^{\text {tz }}$ & $7.315^{x * x}$ & $0.298^{z x z}$ & $0.132^{x z}$ & $0.688^{2}$ & 0,434 & $7.308^{x x z}$ & 0,169 & $-0,0,036$ & $0.289^{*}$ & $0.112^{*}$ & 0,325 & $6.536^{2 \pi x}$ \\
\hline La Libertad & 0,028 & $0.396^{* \pm x}$ & 0,135 & $9.372^{\text {zxx }}$ & 0,029 & $0.365^{\text {tax }}$ & 0,236 & 0,287 & $9.466^{* x *}$ & $-0,019$ & $0.248^{*}$ & $-0.271^{*}$ & $0.437^{* x+2}$ & 0,311 & $9.996^{z * x}$ \\
\hline Lambay & 0,099 & $0.244^{\mathrm{xz} \mathrm{x}}$ & $0.690^{2 x+}$ & $9.186^{x+x}$ & $0.160^{x}$ & $0.221^{\mathrm{xz}}$ & $0.628^{x \neq x}$ & $0.649^{x}$ & $8.614^{x \neq x}$ & 0,266 & $-0,064$ & $-0,067$ & $0.302^{x+x}$ & $0.743^{\text {tx }}$ & $9.214^{\pi x x}$ \\
\hline Lima & $0.117^{2}$ & $0.703^{x \times x}$ & $0.513^{\mathrm{xz}}$ & $9.229^{x x x}$ & $0.168^{z z}$ & $0.660^{x \times z}$ & 0,372 & $0.583^{z}$ & $8.816^{x x k}$ & $-0,145$ & $-0.448^{x z z}$ & $0.144^{2 \pi x}$ & $0.543^{\text {zxz }}$ & $0.452^{\text {zz }}$ & $13.321^{* \pi z}$ \\
\hline Loreto & 0,093 & $0.227^{\star \pi *}$ & 0,076 & $8.771^{\star \star \star x}$ & 0,092 & $0.255^{\text {*kx }}$ & 0,035 & $-0,014$ & $8.770^{* * x}$ & 0,288 & $-0,048$ & 0,011 & $0.199^{\text {** }}$ & 0,033 & $7.944^{* * x}$ \\
\hline Madre de Dios & $-0,010$ & $-0,005$ & $0.76 g^{z=z}$ & $10.458^{x+2}$ & $-0,009$ & $-0,015$ & $0.906^{z x z}$ & $0.525^{*}$ & $10.500^{* x \times}$ & $-0,361$ & $-0.555^{\text {*k }}$ & 0,071 & $0.417^{* x x}$ & $0.375^{*}$ & $16.968^{\text {z*x }}$ \\
\hline Moquegua & $-0,055$ & $0.304^{z * z}$ & $1.279^{* 2 \pi}$ & $11.694^{\star \star * z}$ & 0,009 & $0.281^{* \pi z}$ & 0,786 & $1.069^{\text {tz }}$ & $10.834^{\star \star * x}$ & $-0,308$ & $0.582^{* k}$ & $-0.39^{* *}$ & $0.302^{* * x}$ & $0.889^{\text {tat }}$ & $11.639^{\text {t*k}}$ \\
\hline Pasco & $-0,063$ & $0.069^{\text {** }}$ & 0,040 & $10.293^{* * x}$ & $-0.101^{*}$ & $0.053^{*}$ & 0,026 & $0.607^{*}$ & $10.863^{\star \pm x}$ & 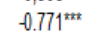 & 0,080 & $0.219^{* *}$ & $0.034^{*}$ & 0,028 & $12.656^{* \pi x}$ \\
\hline Piura & $0.364^{\mathrm{zzx}}$ & 0,058 & $0.911^{\star \pi x}$ & $7.271^{* x *}$ & $0.311^{\text {trtx }}$ & $0.155^{\text {tat }}$ & $0.703^{x}$ & $1.007^{\star *}$ & $7.647^{\mathrm{*x \textrm {x }}}$ & 0.253 & $0.439^{\text {*k }}$ & $-0,191$ & 0,037 & $0.764^{ \pm x *}$ & $6.346^{* * x}$ \\
\hline Puno & $0.327^{* \pi *}$ & $0.214^{ \pm z}$ & $0.343^{x *}$ & $6.558^{x \neq z}$ & $0.339^{2 \pm x}$ & $0.100^{*}$ & $-0,324$ & $0.930^{z * z}$ & $6.107^{7 \pm x}$ & $0.249^{\text {tz }}$ & $0.422^{* z z}$ & $-0.258^{* z *}$ & 0.025 & $-0,148$ & $5.675^{\text {*zx }}$ \\
\hline San Martin & 0,050 & $0.329^{2 \pi x}$ & 0,282 & $9.175^{x * x}$ & 0,117 & $0.270^{x z x}$ & $-0,086$ & $0.838^{\text {tz }}$ & $8.525^{5 * x}$ & 0,337 & 0,083 & $-0.376^{\text {tz }}$ & $0.248^{\text {tx }}$ & $0.323^{x}$ & $9.709^{x+x}$ \\
\hline Tacna & $0.23^{\text {t*x}}$ & $-0,132$ & $0.723^{x+x}$ & $8.239^{ \pm t * t}$ & $0.266^{n+1+}$ & $-0.268^{\text {tztx }}$ & $0.745^{\text {tzxt }}$ & $1.243^{\operatorname{trx}}$ & $7939^{ \pm * x}$ & 0.074 & $0.305^{\text {tx }}$ & -0.242 & $-0,068$ & $0.512^{\text {tt }}$ & $8.955^{t * t}$ \\
\hline Tumbes & $0.336^{z z z}$ & $0.147^{2 z}$ & $0.570^{x *}$ & $6.893^{2 x x}$ & $0.359^{2 x+x}$ & $0.192^{\mathrm{xz}}$ & 0.227 & 0.116 & $6.372^{* x}$ & $-0,193$ & 0.147 & $0.26^{2 z}$ & $0.104^{*}$ & 0,400 & $7.971^{\approx z x}$ \\
\hline Ucayali & $0.129^{*}$ & $0.227^{*}$ & 0,177 & $8.302^{\text {**x }}$ & $0.162^{\text {*z }}$ & 0,192 & $-0,033$ & 0,361 & $7.948^{* \approx *}$ & 0,302 & $-0,094$ & 0,099 & $0.206^{*}$ & 0,174 & $7.343^{2 * x}$ \\
\hline Casos sig. & 15 & 15 & 16 & 23 & 17 & 16 & 9 & 15 & 23 & 4 & 14 & 9 & 16 & 10 & 23 \\
\hline
\end{tabular}

a US\$ 38.515 (Casanaré). En Perú, el VAB per cápita PPA, está entre US\$ 3.278 (Apurímac) y US\$ 29.521 (Moquegua) ${ }^{15}$. Sin embargo, mientras en Colombia 6 de sus 34 departamentos tienen un PIB per cápita PPA de al menos US\$ 14.200 - ingreso per cápita aproximado de los países de ingreso medio alto, según el Banco Mundial -, en Perú sólo Moquegua sobrepasa dicho nivel, siendo que la mayoría de las regiones tienen un nivel de producción comparable con los países de ingreso medio o menor.

Precisamente, el hecho de que los coeficientes del capital en desarrollo social resulten significativos en la mayoría de regiones, exhibiendo valores bastante elevados - en tanto coeficientes del capital en fomento productivo y transporte y comunicaciones son en su mayoría no significativos y, a excepción de pocos casos, presenten valores negativos - va en línea con el "enfoque dual" de Hansen (1965), según el cual en regiones poco desarrolladas se espera que las inversiones en servicios sociales, por ejemplo salud y educación, sean mucho más provechosas que otros tipos de inversión.

Este hallazgo nos hace cuestionar el hecho de que en muchas regiones se le ha otorgado prioridad a la infraestructura vial, o en todo caso no ha existido una priorización y los mayores montos de inversión en transportes son producto de la improvisación, ello en desmedro de otras clases de infraestructura

\footnotetext{
${ }^{15}$ Cálculos propios en base a estadísticas de US Department of Commerce - Bureau of Economic Analisys de EEUU, Departamento Administrativo Nacional de Estadística (DANE) de Colombia, el Instituto Nacional de Estadística e Informática (INEI) de Perú y el Banco Mundial. Todas las cifras son al año 2014.
}

ligada a la acumulación de capital humano.

En lo que se refiere a debilidades institucionales que afectan negativamente el crecimiento de las regiones, es posible establecer algunas: bajo respeto a las leyes; inestabilidad política y social; falta de credibilidad; incentivos mal alineados que dan lugar, en sus versiones más extremas, a corrupción y búsqueda de rentas; entre otros. Así, instituciones de calidad promueven inversiones más productivas, mientras que instituciones débiles generan mayores costos de transacción, conduciendo a una mala asignación, desperdicio de recursos y llevando a un sesgo en la selección de proyectos (Zouhaier, 2012).

La eficiencia y/o calidad de las inversiones públicas ejecutadas, se relaciona con el nivel de exigibilidad del Sistema Nacional de Inversión Pública. En el caso peruano, en el que el SNIP se encuentra descentralizado, este aspecto podría ser importante para explicar las diferencias de productividad entre regiones. Lamentablemente, en la actualidad no se cuenta con indicadores de calidad de inversiones a nivel descentralizado; de hecho, los intentos por hacer mediciones de este tipo a nivel de países aún son exploratorios. Un avance en la materia es la propuesta de Dabla-Norris et al. (2012), quienes proponen un "Índice de eficiencia de la Inversión Pública" enfocado en la dimensión de la gestión de las inversiones, mediante el cual se comparan países. 


\section{Conclusiones}

En línea con el enfoque de la "Nueva Economía Regional", se plantea que la inversión pública incide positivamente en el nivel de producción de las regiones, no obstante la contribución es diferenciada según región. Asimismo, se postula que las inversiones ligadas al desarrollo del capital humano son las que favorecen, en mayor medida, el nivel de producción regional.

La evidencia respalda la existencia de una relación positiva y significativa entre el capital público -que resulta de la acumulación del flujo de inversiones año a año- y el nivel de producción regional - medido por el Valor Agregado Bruto (VAB) de la Producción. El coeficiente obtenido para el capital público per cápita -entre 0,19 y 0,21- indica que la inversión pública juega un papel muy importante en el desempeño económico de las regiones. Además, se encuentra que la causalidad (en el sentido de Granger) es unidireccional en 16 regiones, fluyendo desde el capital público al VAB. También se aporta evidencia a favor de distintas elasticidades producto-capital según región, es decir que el capital público es más productivo en algunas regiones que en otras.

Aunque el estudio no incluye de manera explícita variables relacionadas a la institucionalidad o la eficiencia en las inversiones públicas, estos factores, entre otros, podrían contribuir a explicar las diferencias antes señaladas. Por su parte, el bajo porcentaje de gasto en $\mathrm{I}+\mathrm{D}$ que realiza el Perú en conjunto, y que se diluye a nivel regional, podría ser relevante para explicar el incipiente nivel de desarrollo que presentan todas las regiones a excepción de Moquegua.

Por otro lado, en general, es posible concluir acerca de la importancia de la inversión pública en infraestructura social relacionada a la formación de capital humano. El capital en desarrollo social resulta significativo en 14 casos, con coeficientes positivos que oscilan entre 0,21 (Huancavelica) y 0,68 (Cusco).

Respecto a la contribución del capital en fomento productivo y transporte y comunicaciones al nivel de producción regional, la evidencia no resulta contundente. El coeficiente de fomento productivo solo es significativo en cuatro regiones (Amazonas, Ica, Moquegua y Puno), siendo negativo para todas ellas, excepto en Puno (0,25). Por su parte, el coeficiente del capital en transporte y comuniciaciones resulta significativo en 9 regiones, siendo positivo solo en cuatro de ellas (Junín: 0,29; Lima: 0,14; Pasco: 0,22 y Tumbes, 0,26), esto contrasta con hallazgos previos para el caso peruano en los que se estudia parcialmente ciertos tipos de infraestructura y se concluye a favor de un efecto positivo de la infraestructura en caminos y carreteras, electricidad y telecomunicaciones (Vásquez y Bendezú, 2008; Urrunaga y Aparicio, 2012).

\section{Recomendaciones}

Las implicancias de lo expuesto van en línea con seguir priorizando las inversiones públicas como se ha venido haciendo hasta ahora. Sin embargo, es necesario avanzar hacia el establecimiento de una política de inversiones que priorice la formación del capital humano. La infraestructura para el desarrollo social debe ser generada antes que la infraestructura física o por lo menos simultáneamente. Es importante recordar que las inversiones en infraestructura física y social no son antagónicas sino que se complementan la una a la otra.

Las políticas de inversiones deben, además, ser lo suficientemente flexibles para permitir que las inversiones territoriales vayan acorde con los niveles de desarrollo y con las necesidades diferenciadas de los territorios. Por ello, es sumamente importante fortalecer la capacidad técnica de los gobiernos subnacionales en materia de planificación del desarrollo territorial. De este modo, se recomienda que sean los propios territorios quienes prioricen las inversiones con alto impacto en su desempeño económico y social.

Respecto a la asignación de las inversiones entre regiones, se sugiere mejorar la coordinación intersectorial e intraterritorial. Ello con la finalidad de que el gobierno nacional (que tiene mayor poder de discrecionalidad respecto a la asignación de los montos de inversión según territorio) no continúe exacerbando la inequidad presente de por sí debido a situaciones más estructurales, como la asignación de recursos del canon y regalías, lo cual por Ley se realiza bajo un enfoque de localización de los recursos.

Finalmente, se recomienda futuros estudios que permitan identificar los aspectos institucionales relevantes para el crecimiento de las regiones de Perú, así como profundizar en la contribución de otras variables como la eficiencia y/o calidad de las inversiones y la ciencia, tecnología e innovación. Ello implica, desde luego, avanzar en la compilación de estadísticas descentralizadas que permitan medir adecuadamente estos aspectos. 


\section{Referencias}

Agrawal, P. (2007). Economic growth and poverty reduction: evidence from kazakhstan. Asian Development Review, 24(2):90.

Alexiadis, S. (2012). Convergence clubs and spatial externalities: Models and applications of regional convergence in Europe. Springer Science \& Business Media.

Arellano, M. y Bond, S. (1991). Some tests of specification for panel data: Monte carlo evidence and an application to employment equations. The review of economic studies, 58(2):277-297.

Arellano, M. y Bover, O. (1995). Another look at the instrumental variable estimation of errorcomponents models. Journal of econometrics, $68(1): 29-51$.

Arrow, K. (1962). Economic welfare and the allocation of resources for invention. En The rate and direction of inventive activity: Economic and social factors, pp. 609-626. Princeton University Press.

Aschauer, D. A. (1989a). Does public capital crowd out private capital? Journal of monetary economics, 24(2):171-188.

Aschauer, D. A. (1989b). Is public expenditure productive? Journal of monetary economics, 23(2):177-200.

Banco Mundial (2013). La Formulación de Políticas en la OCDE: Ideas para America Latina.

Barro, R. J. y Sala-i Martin, X. (1990). Economic growth and convergence across the united state. NBER Working paper, (3419).

Bertoni, R., Castelnovo, C., Cuello, A., Fleitas, S., Pera, S., Rodríguez, J., y Rumeau, D. (2011). ¿Qué es el desarrollo? ¿Cómo se produce? ¿Qué se puede hacer para promoverlo? Construcción y análisis de problemas del desarrollo. Universidad de la República.

Bhagwati, J. y Srinivasan, T. N. (2002). Trade and poverty in the poor countries. The American Economic Review, 92(2):180-183.

Button, K. (1998). Infrastructure investment, endogenous growth and economic convergence. The annals of regional science, 32(1):145-162.

Casey, S. (2005). Establishing standards for social infrastructure. UQ Boilerhouse, Community Engagement Centre.

Cass, D. (1965). Optimum growth in an aggregative model of capital accumulation. The Review of economic studies, 32(3):233-240.
Change, E. T. (1990). Endogenous technological change. journal of Political Economy, 98(5):2.

Cobacho, M., Bosch, M., y Rodríguez, E. (2004). Efectos de la inversión pública federal en méxico. Anales de Economía Aplicada.

Dabla-Norris, E., Brumby, J., Kyobe, A., Mills, Z., y Papageorgiou, C. (2012). Investing in public investment: an index of public investment efficiency. Journal of Economic Growth, 17(3):235266.

Dawkins, C. J. (2003). Regional development theory: conceptual foundations, classic works, and recent developments. Journal of planning literature, 18(2):131-172.

Deaton, A. y Dreze, J. (2001). Trade, growth, and poverty. Finance and Development, 38:16-9.

Demurger, S. (2001). Infrastructure development and economic growth: an explanation for regional disparities in china? Journal of Comparative economics, 29(1):95-117.

Dollar, D. y Kraay, A. (2003). Institutions, trade, and growth. Journal of monetary economics, 50(1):133-162.

Domar, E. D. (1946). Capital expansion, rate of growth, and employment. Econometrica, Journal of the Econometric Society, pp. 137-147.

Drezgić, S. (2008). The effects of public sector investments on economic growth of croatia. En Young Economist's Seminar to 14th Dubrovnik Economic Conference: Proceedings Zagreb: Croatian National Bank, pp. 1-49.

González Páramo, J. M. y López, D. M. (2003). Public investment and convergence in the spanish regions. En Hacienda pública y convergencia europea: X Encuentro de Economía Pública, Santa Cruz de Tenerife 2003, p. 52. Universidad de La Laguna.

Gramlich, E. M. (1994). Infrastructure investment: A review essay. Journal of economic literature, 32(3):1176-1196.

Harrod, R. F. (1939). An essay in dynamic theory. The economic journal, 49(193):14-33.

Hernández, J. L. (2010). Inversión pública y crecimiento económico: Hacia una nueva perspectiva de la función del gobierno. Economía: teoría y práctica, (33):59-95.

Mäki-Arvela, P. (2002). Testing the neoclassical model of regional economic growth: A panel data approach across the finnish provinces, 1973-1996. The Review of Regional Studies, 32(2):223. 
Mendoza Tolosa, H. A. y Yanes Guerra, C. A. (2014). Impacto del gasto público en la dinámica económica regional. Revista Finanzas y Política Económica, 6(1):23-41.

Moody, C. E. (1974). The measurement of capital services by electrical energy. Oxford Bulletin of Economics and Statistics, 36(1):45-52.

Munoz, E. y Vindas, K. (1995). Pruebas extendidas de granger. Banco Central de Costa Rica, División Económica.

Noriega, F. (1965). On the concept of optimal economic growth.

Noriega, F. (2011). Crecimiento exógeno y endógeno: Bases del debate. pp. 93-112.

PNUD (2010). Informe desarrollo humano 2010: Caminos al desarrollo humano.

PNUD (2013). Informe desarrollo humano en perú 2013: Cambio climático y territorio: Desafíos y respuestas para un futuro sostenible.

Ponce, E. (2013). Inversión pública y desarrollo económico regional.

Ramsey, F. P. (1928). A mathematical theory of saving. The economic journal, 38(152):543-559.

Ravallion, M. y Datt, G. (2002). Why has economic growth been more pro-poor in some states of india than others? Journal of development economics, 68(2):381-400.

Richardson, H. W. (1979). Aggregate efficiency and interregional equity. En Folmer, H., editor, Spatial inequalities and regional development, pp. 161-183. Springer.

Romer, P. M. (1986). Increasing returns and longrun growth. The journal of political economy, pp. 1002-1037.

Shi, Y. (2012). The Role of Infrastructure Capital in China's Regional Economic Growth. Manuscript, Michigan State University.
Solow, R. M. (1956). A contribution to the theory of economic growth. The quarterly journal of economics, pp. 65-94.

Stough, R. R., Stimson, R. J., y Nijkamp, P. (2011). An endogenous perspective on regional development and growth. En Drivers of Innovation, Entrepreneurship and Regional Dynamics, pp. 3-20. Springer.

Straub, S. (2008). Infrastructure and growth in developing countries, volumen 4460. World Bank Publications.

Swan, T. W. (1956). Economic growth and capital accumulation. Economic record, 32(2):334-361.

Urrunaga, R. y Aparicio, C. (2012). Infraestructura y crecimiento económico en el perú. Revista Cepal.

Uzbay, M. y Lenger, A. (2011). The role of public capital in regional economy. En International Workshop on Regional Competitiveness and International Factor Movements, Laboratoire d'Economie d'Orleans-Network for Economic Research INFER, France.

Vásquez, A. y Bendezú, L. (2008). Ensayo sobre el rol de la infraestructura vial en el crecimiento económico del perú. En Ensayo sobre el rol de la infraestructura vial en el crecimiento económico del Perú. Consorcio de Investigación Económica y Social.

Wenjun, F. y Jing, Z. (2011). The empirical analysis on relationship between infrastructure investment and regional economic growth in china. En 8th International Conference on Innovation $\&$ Management, pp. 349-353.

Zouhaier, H. (2012). Institutions, investment and economic growth. International Journal of Economics and Finance, 4(2):152. 